\title{
Emotional expressivity in men and women: Stereotypes and self-perceptions
}

\author{
Ursula Hess and Sacha Senécal \\ University of Quebec at Montreal, Canada \\ Gilles Kirouac \\ Laval University, Quebec, Canada \\ Pedro Herrera \\ University of Quebec at Montreal, Canada \\ Pierre Philippot \\ University of Louvain-La-Neuve, Belgium \\ Robert E. Kleck \\ Dartmouth College, Hanover, NH, USA
}

\begin{abstract}
Three studies were conducted to assess prevalent stereotypes regarding men's and women's emotional expressivity as well as self-perceptions of their emotional behaviour. Emotion profiles were employed to assess both modal emotional reactions and secondary emotional reactions to hypothetical events and personal experiences. In Study 1 we asked how men and women in general would react to a series of hypothetical emotional events. In Study 2 we asked how participants themselves expected to react to these same situations and in Study 3 we asked participants to report a personal emotional event in narrative form. Two gender differences emerged across all three studies. Specifically, women were expected to be more likely to react with sadness to negative emotion-eliciting events in general. They also expected themselves to be more likely to react with sadness as well as to cry and to withdraw more when experiencing negative emotional events. Finally, women report more sadness when describing personal events. In contrast, men were expected to react with more happiness/serenity during negative emotional situations. Also, they expect themselves to react more frequently this way as well as to laugh and smile more and to be more relaxed in negative
\end{abstract}

Please send correspondenc e and requests for reprints to Ursula Hess, Department of Psychology, University of Quebec at Montreal, P.O. Box 8888, Station A, Montreal, Qc H3C 3P8, Canada.

This research was funded by grant from the Conseil de Recherche en Sciences Humaines to the first and fourth author. The authors wish to thank Nathalie Houlfort, Nathalie Collette, Valérie Vézina, Pascal Thibault, and Maxime Lévesque for their help in conducting the research described. 
situations. Finally, men tend to report more happiness when describing negative personal events. In sum, the present study gives a more detailed portrayal of how men and women are expected and expect themselves to react to specific emotional situations and presents some evidence that these expectations may influence the way they reconstruct emotional events from their past.

One of the most consistent empirical findings in the current literature on sex differences regards emotional expressivity. Differences in emotional expressivity between men and women are found when considering self-reports of emotional expressiveness, when using observational studies, as well as when considering beliefs and stereotypes about emotional expressiveness. The present research investigates beliefs and stereotypes concerning emotional expressivity and investigates to what degree these beliefs and stereotypes overlap with selfperceptions of emotional expressivity, as well as with personal narratives involving emotional events.

Stereotypical beliefs regarding emotions are culture-specific and reflect shared notions regarding the prevalence of certain feelings and behaviours experienced and expressed by men and women. At the same time, these stereotypes can also influence self-reports of emotional events based on autobiographic memory. For example, Sherman and Bessenhoff (1999) provide convincing evidence of stereotype influences on source attributions in autobiographic memory under conditions of limited processing capacity. Also, Feldman-Barrett, Robin, Pietromonaco, and Eysell (1998) suggest that global self-reports of emotional expressivity are influenced by cultural beliefs as individuals have to summarize over many types of events from their past - a process that may lead to the use of heuristics that bias recollections in favor of culturally shared beliefs. Yet, accounts of emotional events constitute a significant part of our daily social exchanges with friends and family members as research on emotional sharing demonstrates (e.g., Rimé, Mesquita, Philippot, \& Boca, 1991). This leads to the speculation that some accounts of events that happened to us in the past and such statements as "I am one of those people who often cry when watching sad films", which make up a significant part of our social exchanges, may reflect our culturally shared beliefs as well as actual events.

\section{Gender stereotypes of emotional expressivity}

In Western cultures women are believed to be more emotionally expressive in general than are men. Specifically, they are expected to smile more as well as to show more sadness, fear, and guilt. In contrast, men are believed to show more overt emotional displays only in terms of physically aggressive anger (for reviews see Brody \& Hall, 1993; Fischer, 1993). These gender stereotypes appear to be socialised into children's belief systems as early as 3-5 years (Birnbaum, 1983). Studies on the parental socialisation of emotions suggest that 
both mothers and fathers use more varied emotion terms, and more of them, when talking to daughters than when talking to sons. Further, some emotions, such as sadness, are mentioned more often to daughters than to sons (Adams, Kuebli, Boyle, \& Fivush, 1995; Dunn, Bretherton, \& Munn, 1987; Fivush, 1989). These socialisation practices are obviously consistent with a belief on the part of parents that females should be more interested in emotion as well as expressive of it than should males. To the extent that this belief of greater emotional expressivity in females reflects actual behavioural differences across the genders we would obviously be hesitant to label it a stereotype, though a stereotypical belief could ultimately be the source of the differences. Whereas those studies that have examined observed expressivity in minimal social situations (noninteractive), found that females tend to be somewhat more expressive than are males (see e.g., Fischer, 1993), studies that have examined actual male versus female expressivity in a larger context paint a relatively more complex picture (see e.g., Kring \& Gordon, 1998).

Yet, although the general gender stereotype and global self-descriptions suggest a large difference in expressivity between men and women, observed differences and self-reports of specific events yield little or no gender effects. For example, Feldman-Barrett et al. (1998) found sex-related differences for global self-descriptions of emotions but not for ratings obtained in a diary study where participants rated their emotions following social interactions. In a similar vein, Jussim, Milbourn, and Nelson (1991), who used telephone interviews to assess self-perceptions of emotional expressivity as well as perceptions of emotional expressivity in men and women in general, found that although both men and women believed that women are more emotionally expressive than men, there were little or no differences in self-perceptions. This effect was stronger for older participants than for younger participants. Jussim et al. (1991) interpret this finding as support for a self-fulfilling prophecy effect of stereotypes on actual behaviour.

An exception regards smiling behaviour that is both believed and observed to be more typical for women and aggressive anger reactions which are found to be more typical for men (see Briton \& Hall, 1995; Brody \& Hall, 1993; Fischer, 1993). However, despite these exceptions, evidence is accumulating that observed emotional expressivity depends on the details of the social situation as well as on personality factors as has been underlined in previous research (Feldman-Barrett, et al., 1998; Fischer, 1993; Hall, 1984; LaFrance \& Banaji, 1992; Tucker \& Riggio, 1988).

Thus, when studying stereotypes and self-perceptions of emotional expressivity we need to distinguish three different levels. First, beliefs regarding the emotional behaviour of men and women in general. Second, selfdescriptions by men and women of emotional reactions to certain types of specific emotion-inducing events (e.g., learning that a supposed friend is spreading unpleasant rumours) and finally, men's and women's autobiographic 
narratives of a specific emotion event. All three types of reports should reflect the influence of cultural beliefs regarding emotional expressivity but to different degrees. The level of similarity between these different reports should help us better understand the prevalence and influence of these beliefs for situations in which people report emotions. Further, as Jussim et al. (1991) suggest, describing one's emotional experiences in terms of culturally shared beliefs about the nature of these experiences may, in the long run, lead to changes in the way events are experienced.

\section{"Pure" emotions vs. emotion profiles}

The body of research on gender stereotypes for emotions briefly reviewed earlier, is based primarily on studies that focus either on "pure" emotional states such as in studies on the self-reported frequency of experience of certain emotions, on beliefs regarding the frequency with which these emotions are experienced, or on certain behavioural modes for such pure states as in studies on crying when angry.

Yet, "pure" emotional states are rare and most emotional situations elicit more than one emotion, with some being more prominent than others (Izard, 1972; Plutchik, 1980). An interesting implication of this is that gender stereotypes regarding differences in emotional expressiveness may not only involve differences in the expression of a principal emotion (e.g., sadness in response to an irrevocable loss) but also differences regarding the presence of secondary emotions, such as anger or guilt. The presence or absence of these secondary emotions in a particular context is likely to have important implications for how individuals cope with a given event. For example, an assertive expression of "self-righteous" anger may not be possible when feelings of guilt or shame occur at the same time. This suggests that a study on emotional stereotypes should focus not only on "core" emotions but should include an analysis of the secondary emotions that are expected to be elicited in men and women as well.

\section{Overview}

Three studies were conducted. The first assessed emotional reactions of men and women to various emotion-eliciting events. Specifically, respondents were asked to indicate the percentage of women and men who would react with each of nine emotions to a series of short vignettes. Study 2 assessed the level to which participants' self-perceptions of their emotional reactions paralleled the general stereotypes found in Study 1. For this, a subset of the vignettes used in Study 1 were employed. The participants' task was to indicate for each event the likelihood, as a percentage, that they personally would react with each of the nine emotions from the emotion profile when they were confronted with the situations described in the vignettes. Finally, in Study 3, participants were asked 
to describe an event that had happened to them in the past and to indicate the emotions they had felt during the event.

\section{STUDY 1}

The goal of Study 1 was to assess the beliefs held by young adults regarding the emotions likely to be expressed by men and women in specific emotion eliciting situations. Unlike previous research, we were interested in assessing these beliefs on a "profile" measure of expressivity. That is, we wanted to discover not just what individuals perceived to be the modal emotion expressed by males and females in any given situation, but also their views regarding the probability that any of several other basic emotions would be expressed in that situation. Consistent with previous research, we expected that women would be perceived to be more expressive of all emotions than would men, with the exception of anger. Further, we anticipated that the profile measure would reveal consistent gender differences in the secondary, or nonmodal, emotional responses associated with particular eliciting events.

\section{Method}

Materials. The emotion scenarios were taken from a large database of selfreported emotion eliciting events (ISEAR, International Survey on Emotion Antecedents and Reactions). ${ }^{1}$ This database contains self-descriptions of emotion related events from 2921 participants from 37 countries. For each of seven basic emotions, events that were frequently reported as involving this emotion were selected from the database. These descriptions were translated into French and any reference to the gender of the protagonist was replaced with a gender neutral noun or preposition.

Four men and four women then estimated, on 7-point scales, to what extent each event represented each of seven core relational themes. Core relational themes (Lazarus, 1991) refer to the common content of events likely to elicit a certain emotion (e.g., for sadness the core relational theme involves an irrevocable loss). A 7-point scale regarding the social versus individual character of each vignette was also completed by the participants in this pre-test. Using these data, four vignettes for which the target emotional theme was identified and which were perceived as describing social situations,

\footnotetext{
${ }^{1}$ The data are part of an international project ISEAR (International Survey on Emotion Antecedents and Reactions) initiated by Klaus Scherer, University of Geneva. The development of the databank has been supported by the Maison des Sciences de l'Homme (Paris, France), the Thyssen Foundation (Germany), and the Société Académique de l'Université de Genève (Switzerland). The data bank consortium consists of Agneta Fischer (University of Amsterdam), Pierre Philippot (University of Louvain at Louvain-la-Neuve), and Harald Wallbott (University of Salzburg). Readers interested in the databank should contact Harald Wallbot: e-mail wallbotthara@edvz.sbg.ac.at or fax: 43066280445126.
} 
were chosen for each of seven basic emotions (happiness, sadness, fear, disgust, anger, shame, and guilt). The following is an example of a guilt vignette: "Someone does not fully participate in a team effort which leads to a bad result for everyone on the team"'. An example of an anger vignette is: "Someone learns that somebody close to him has been spreading negative rumours about him". (The complete set of 28 vignettes is available from the first author).

Dependent measures. Participants were asked to indicate for nine emotions (happiness, serenity, sadness, fear, disgust, contempt, anger, shame, and guilt), the percentage of men and women, as well as the percentage of Anglophones and Francophones, ${ }^{2}$ who would react by expressing each of these emotions in the situation described in the vignette. Although the stimulus events were selected to involve only seven of these basic emotions, "serenity" was added to the rating scale to provide a second positive affective state that could be viewed as a different level of happiness. "Contempt'" was included because some investigators argue that it is separable from disgust, though often confused with it (Ekman \& Friesen, 1986).

Participants. A total of 544 male and female participants were recruited from junior colleges in a suburb of a large urban centre and in a small town in a rural area of Quebec. Table 1 shows the number of subjects for each condition and location.

Procedure. Participants completed the questionnaire in various sized mixed-sex groups. They were told that it assesses the types of emotional reactions that we generally expect from members of various social groups. They were informed of their right to discontinue participation at any time and for any reason. The experimenter then distributed the questionnaires, explained the use of the scales, and responded to any questions regarding the procedure. To limit the duration of the experiment to approximately 30 minutes so as to avoid fatigue, each questionnaire contained only 8 or 12 of the 28 vignettes. The vignettes were arbitrarily divided into three sets for presentation to the participants: those describing anger and disgust situations, those for sadness and fear situations, and finally, those for guilt and shame situations. For a third of the participants, the four happiness vignettes were included in the set of situations they responded to.

Data reduction and analyses. The ratings for the four vignettes associated with each specific emotion were averaged. All analyses were conducted on these

${ }^{2}$ The last two ratings served as distractors for this study and were not included in the analyses (see Eagly \& Mladinic, 1989, for a similar approach). 
TABLE 1

Number, sex, and location of participants as a function of condition (Study 1)

\begin{tabular}{lrrr}
\hline & \multicolumn{2}{c}{ Number of subjects } & \\
\cline { 2 - 3 } Condition & Men & Women & Total \\
\hline Anger-Disgust & & & \\
$\quad$ Urban & 55 & 37 & 92 \\
Rural & 31 & 58 & 89 \\
Total & 86 & 95 & 181 \\
Shame-Guilt & & & \\
Urban & 35 & 56 & 91 \\
Rural & 32 & 56 & 88 \\
Total & 67 & 112 & 179 \\
Sadness-Fear & & & \\
$\quad$ Urban & 55 & 43 & 98 \\
$\quad$ Rural & 23 & 56 & 86 \\
Total & 78 & 106 & 184 \\
Happiness & & & \\
Urban & 75 & 56 & 141 \\
Rural & 45 & 87 & 132 \\
Total & 120 & 143 & 263 \\
\hline
\end{tabular}

means. To assess differences in expected emotional reactions for men and women, profile analyses were employed. Profile analyses permit us to assess whether emotion ratings varied as a function of the sex of the protagonist. If men and women are perceived to react differently, then the profiles will be nonparallel. If they are expected to react in a similar manner, even though possibly at different overall levels, the profiles will be parallel. Differences in the overall level of emotional responding can be diagnosed by assessing whether the profiles are coincident.

\section{Results}

Sex of rater. The emotion expression profiles for male and female participants were compared for each of the seven emotion situations separately for the rural and the urban sample and for men's and women's expected reactions. The results from 26 of the resulting 28 profile analyses show that the profiles are parallel and coincident. That is, male and female participants' judgements described the same emotion profiles with comparable levels for all emotions. The data from male and female participants were therefore collapsed for all further analyses. 
Emotion profiles. As expected, distinct profiles emerged for each of the emotion themes (see Figures 1a-g). Further, the profiles describing men's and women's perceived reactions were not parallel (see later); however, the profiles were generally similar with regard to their major features. As the focus of the present paper concerns sex differences, we will restrict the discussion of the overall form of the profiles to two general observations. First, the profiles for all negative emotion situations show a prominent peak for the target emotion as well as secondary peaks. That is, the perceived reactions to the emotional events used here typically involved both secondary emotions as well as a modal emotion. It should be noted that an inspection of the raw data suggests that the peaks for both modal and secondary emotions are reflected in the individual ratings and are not an artefact of averaging over divergent ratings. Put another way, the derived "average", profiles reflect those obtained from individual perceivers.

As Figure 1 shows, anger, sadness, and contempt were frequently expected to co-occur. Further, participants expected similar levels of shame and guilt for vignettes involving either of these specific emotions. This suggests that these two emotions were not well differentiated by the participants; a finding that is in accordance with the notion that in Western cultures shame and guilt are not as separate as is the case in some other cultures (see e.g., Wallbott \& Scherer, 1994). Second, the profile for the happiness-eliciting events was characterised by the absence of any other expected emotion, with the exception of a small percentage of occasions where serenity was also anticipated. This suggests that happiness was expected to occur as a relatively "pure" emotion. However, as a caveat one should note that only two positive emotions (happiness and serenity) were included in the list of possible emotional reactions and that serenity can be seen as a level of happiness rather than as an emotion distinct from it.

Perceived sex differences. To assess whether the profiles for men's and women's expected reactions to the different emotion eliciting situations were parallel and coincident, profile analyses were conducted on the mean ratings for the four vignettes representing each emotion. These analyses were conducted separately for the rural and urban samples. However, the general findings for the two samples are identical and most post-hoc comparisons that reach significance in one sample do so in the other. Therefore, to facilitate the visual presentation of the data, Figure 1 shows the data combined over the two samples. However, the $F$ values for the analyses are presented in Table 2 separately for the two samples. All analyses and post-hoc tests were conducted with an alpha level of .05.

For all emotion situations except shame and happiness, profiles for men's and women's expected reactions were nonparallel for both samples (see Table 2). For shame vignettes, the two profiles from the urban sample were parallel whereas for happiness the profiles were parallel for both the urban and rural groups. 
(a)

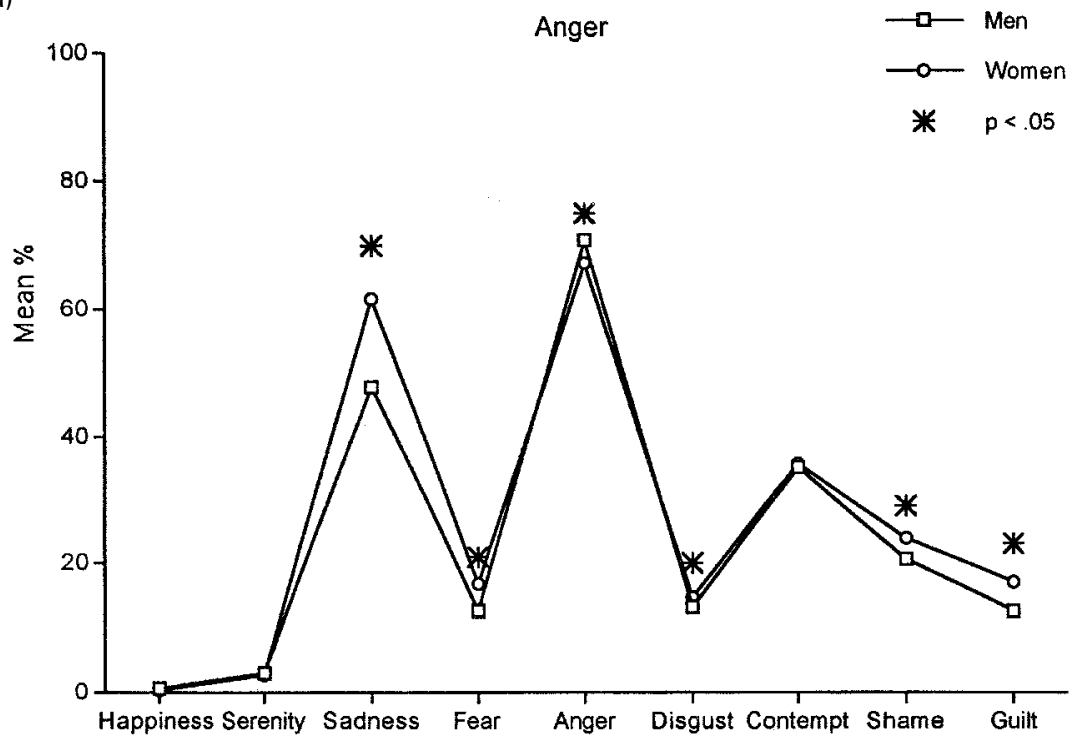

Emotional reaction

(b)

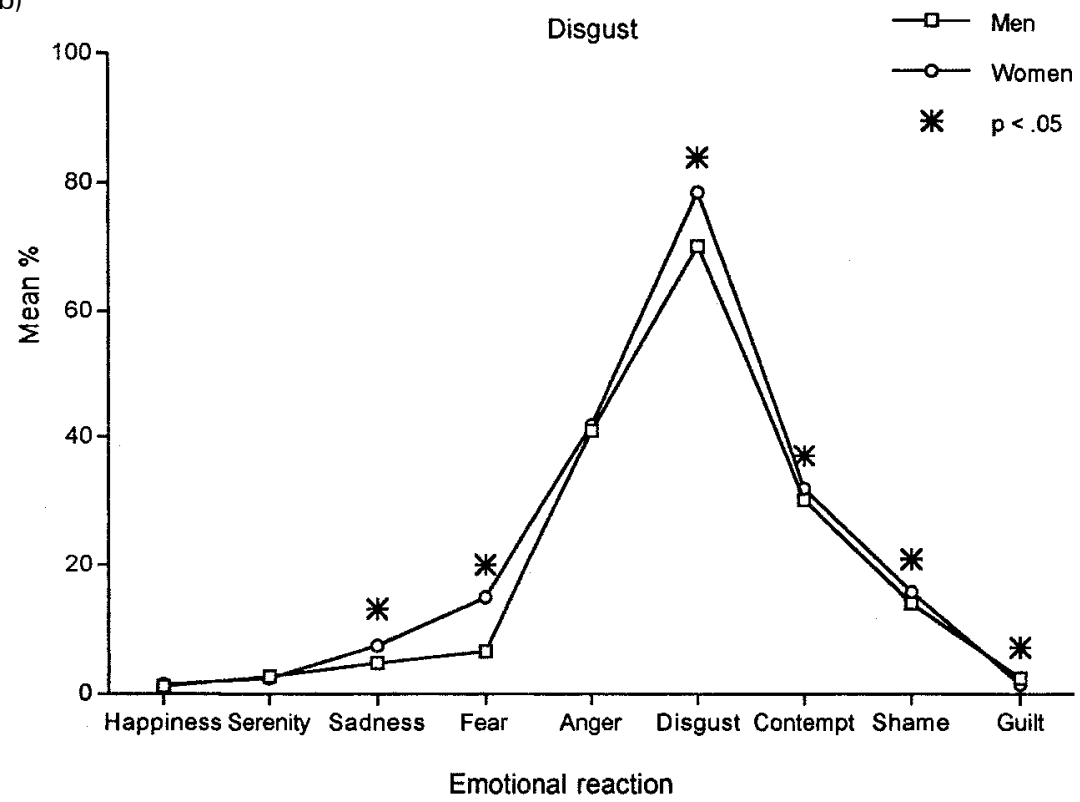

Figure 1. Emotion profiles for men's and women's expected emotional reaction as a function of emotion theme (Study 1).

(Continued overleaf) 
(c)

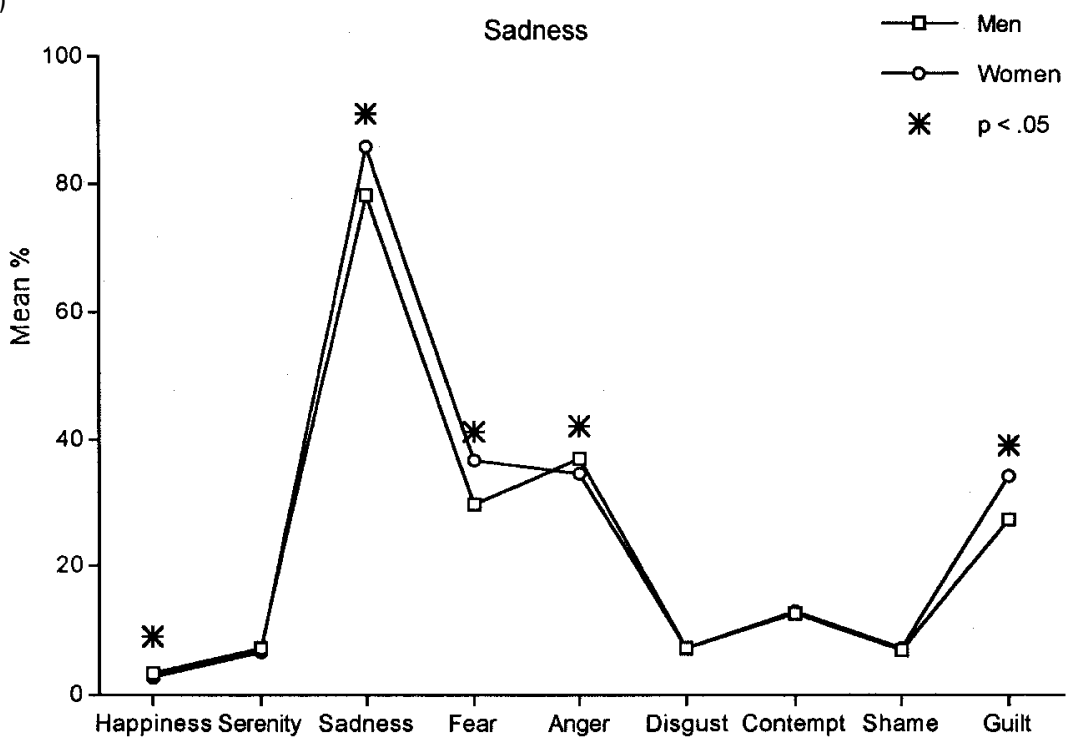

Emotional reaction

(d)

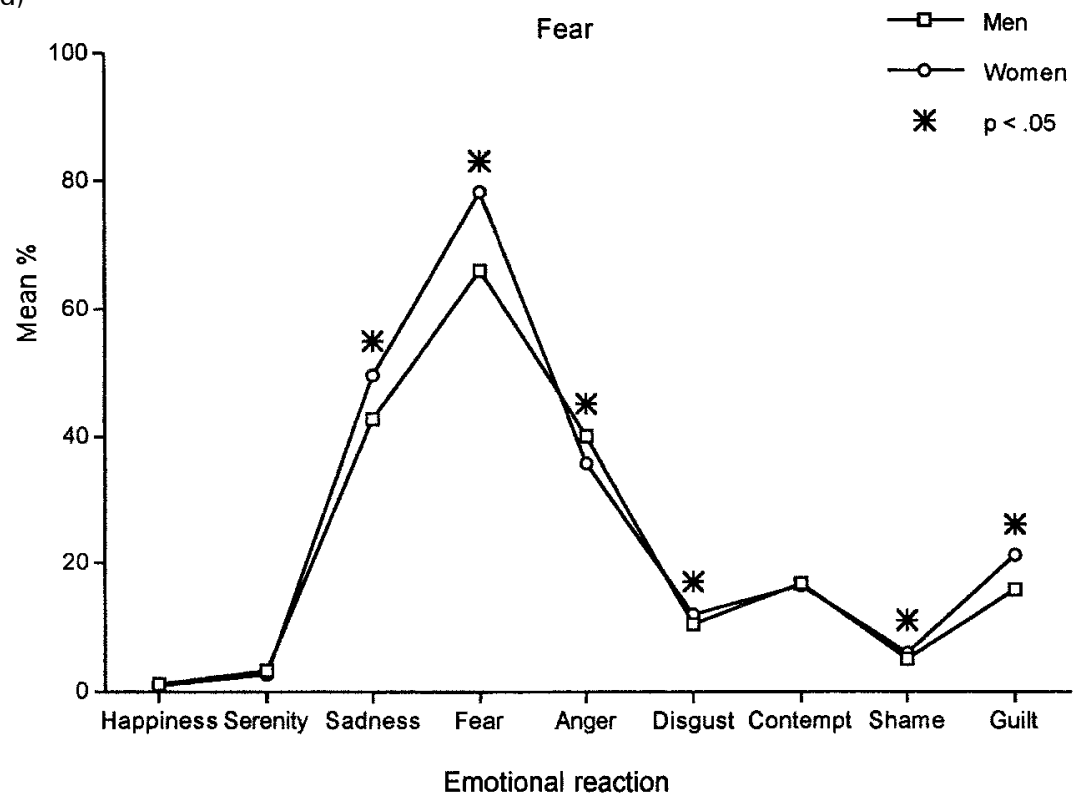

Figure 1. (Continued) 
(e)

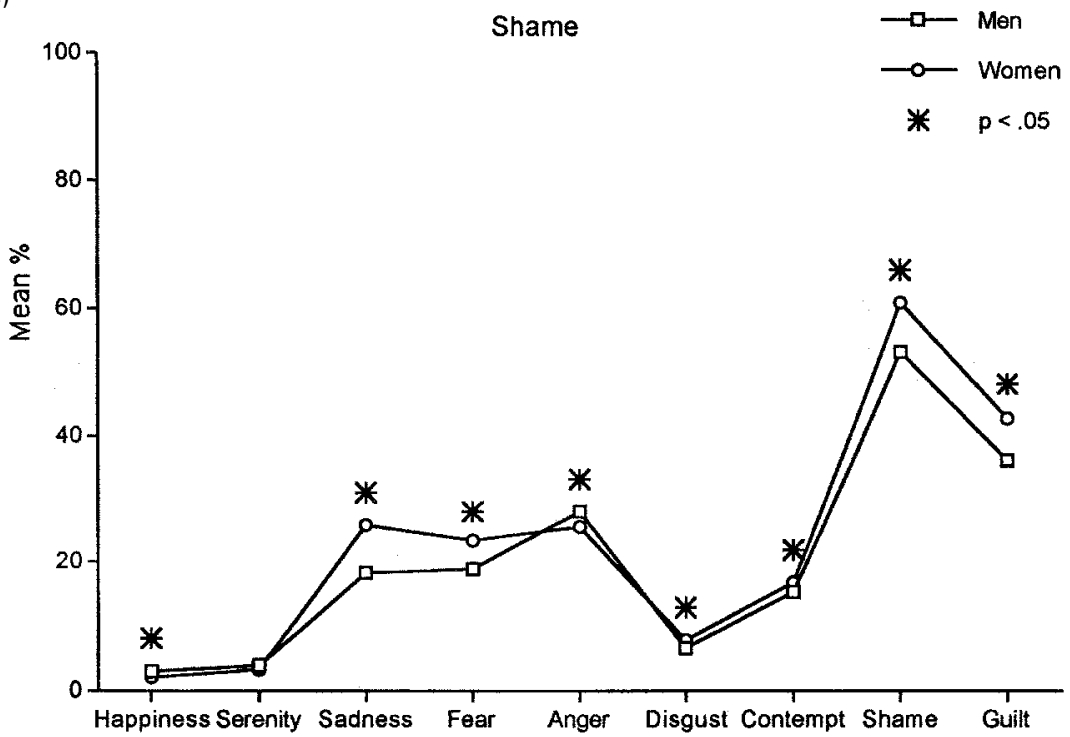

Emotional reaction

(f)

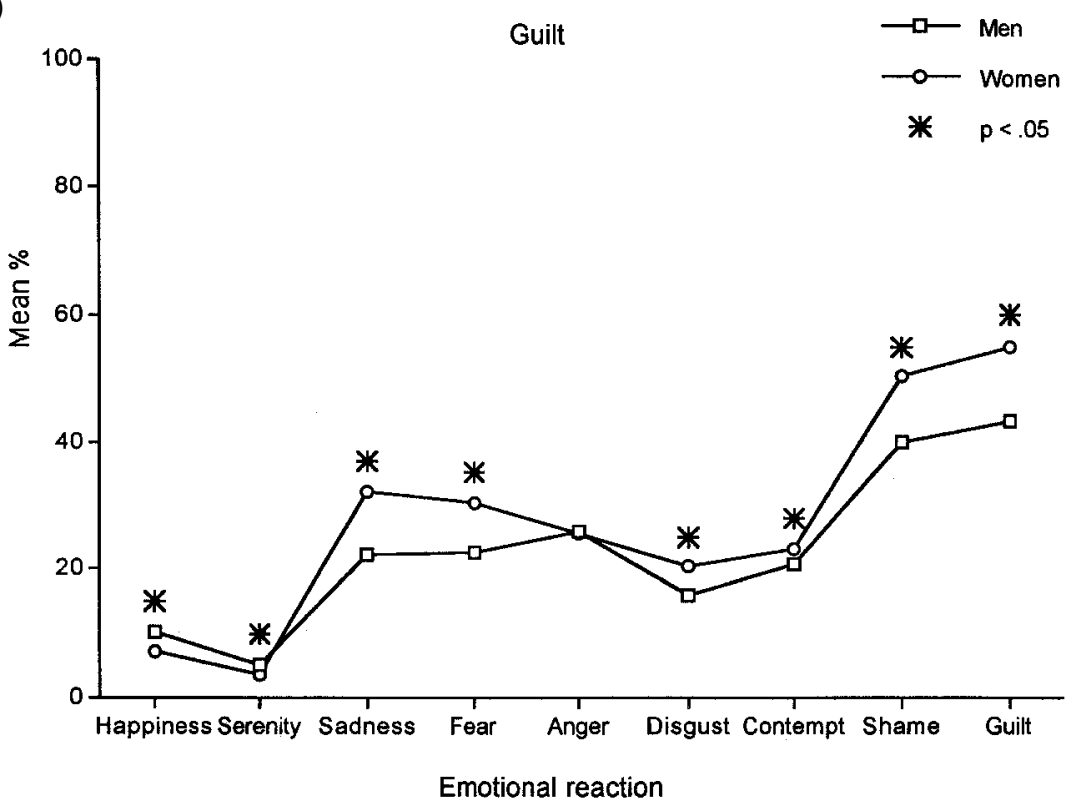

Figure 1. (Continued)

(Continued overleaf) 


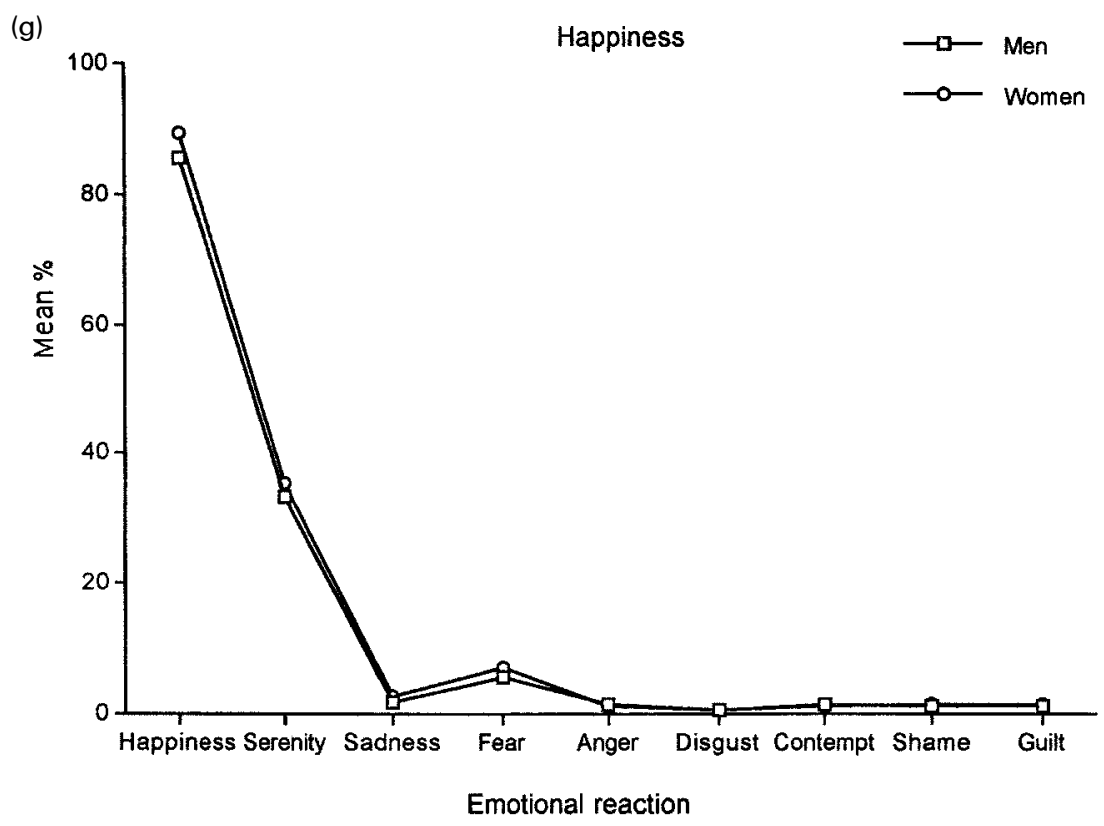

Figure 1. (Continued)

In general, the same differences between men's and women's expected emotional reactions were found for all negative emotion vignettes. Regardless of the central emotion theme of the vignettes, univariate post-hoc tests on the participants' ratings of expected anger, happiness, and serenity, either revealed no differences in expected reactions between men and women, or they revealed that a higher percentage of men than women were expected to react with these emotion displays. Men were judged to be significantly more likely to express anger in sadness, anger, shame, and fear situations. Further, men were judged to be significantly more likely than women to express happiness in guilt, shame, and sadness situations and to express serenity in guilt situations.

On the other hand, a different pattern emerged for participants' ratings of expected sadness, fear, disgust, contempt, shame, and guilt. For these emotional reactions differences emerged for almost all negative emotion situations and in all cases women were expected to be more likely to react with these emotions than were men. Specifically, univariate post-hoc analyses revealed that women were judged to be significantly more likely to react with sadness, fear, and guilt in all emotion-relevant situations. Further, women were judged to be significantly more likely to react with disgust and shame in all emotion situations except for sadness situations. Finally, women were judged to be significantly more likely than men to react with contempt in disgust, guilt, and 
TABLE 2

Results of profile analyses on expected emotional reactions for men and women in general (Study 1)

\begin{tabular}{lccc}
\hline Vignettes & $F$ & $d f$ & $p$ \\
\hline Anger & & & \\
Urban & 5.32 & 8,175 & .001 \\
Rural & 3.33 & 8,169 & .001 \\
Disgust & & & \\
Urban & 5.07 & 8,175 & .001 \\
$\quad$ Rural & 4.61 & 8,169 & .001 \\
Fear & & & \\
Urban & 6.89 & 8,187 & .001 \\
Rural & 2.94 & 8,163 & .004 \\
Sadness & & & \\
Urban & 4.89 & 8,187 & .001 \\
Rural & 2.67 & 8,163 & .009 \\
Guilt & & & \\
Urban & 4.14 & 8,173 & .001 \\
Rural & 2.88 & 8,167 & .005 \\
Shame & & & \\
Urban & 2.77 & 8,173 & .007 \\
Rural & 1.56 & 8,167 & .140 (n.s.) \\
Happiness & & & .090 (n.s.) \\
Urban & 1.74 & 8,273 & .257 (n.s.) \\
Rural & 1.27 & 8,255 & \\
\hline
\end{tabular}

shame situations. For happiness, the profiles for both genders were parallel and coincident, suggesting that raters not only expected the same pattern of reactions from men and women but also at the same level for each emotion.

\section{Discussion}

In summary, the analyses of the emotion profiles suggest the presence of clear expectancies regarding not only the core emotion for a given type of situation, but also for the secondary emotional reactions that are expected in the situation. Although the profiles for men and women are relatively similar with regard to their major features, a number of systematic differences in expectations regarding men's and women's emotional reactions emerged for the negative emotion events but not for the positive.

This latter finding is in contrast to the literature on the self-reported frequency of happiness expressions/experiences in men and women (e.g., Allen $\&$ Haccoun, 1976) and with the finding that women are both believed and 
observed to smile more than men (Briton \& Hall, 1995). However, given the multiple functions of smiles (see e.g., Fernandez-Dols, \& Ruiz-Belda, 1995; Frank \& Ekman, 1993; LaFrance \& Hecht, 1995) differences in smiling may not reflect differences in happiness. One explanation for this lack of continuity with the existing literature is that the results of the present study are based on specific vignettes and not on global recollections. Thus, possible differences in realworld exposure to happiness-eliciting situations are less likely to influence our findings than they are to influence more global assessments.

For all negative emotion events the profiles were nonparallel, ${ }^{3}$ that is, the ratings revealed different expectations for men and women. The differences are consistent with the notion that the gender stereotype for emotional expressiveness does not assume women to be more expressive for all emotions. For example, women are not expected to express more disgust and shame in sadness situations than are men. Also, women are expected to react with more contempt than are men only in disgust, guilt, and shame situations while for all other situations no differences emerge. However, women are thought to express more sadness, fear, shame, and guilt for all emotion-eliciting situations except for happiness events. That is, regardless of the emotion theme prevalent in the vignettes, women are always expected to react with more sadness, fear, shame, and guilt.

An interesting case regards happiness as a secondary emotion in situations with a negative core emotion. The data reveal that men are typically expected to experience more happiness in these situations than are women. The differences are strongest for guilt and shame vignettes, which involve notions of cheating and public misbehaviour, and might reflect a difference in value structure inherent in the gender stereotype.

In sum, the results of Study 1 expand our knowledge regarding gender differences in emotion stereotypes by considering secondary emotional responses as well as modal emotional responses. Specifically, women are not only expected to react with more sadness, fear, shame, and guilt in situations for which these emotions are central, but are expected to do so in all situations involving negative affect. Men, on the other hand, are expected to react with more anger in a wide variety of situations involving negative affect as well as with more happiness in some situations involving negative affect. This suggests that the prevalent emotion stereotype describes women's predominant emotional reactions to be more withdrawing (fear and sadness) and self-directed (shame and guilt), whereas men's emotional reactions are seen as more active and aggressive.

But to what degree are these general stereotypes/expectations reflected in the self-perceptions that people have of their own emotional reactions? As Jussim et al.'s (1991) findings show, self-perceptions can diverge from the cultural stereotypes. Yet, Jussim et al. also suggest that over time these

\footnotetext{
${ }^{3}$ An exception are the shame profiles for the rural sample.
} 
stereotypical beliefs may act as a sort of self-fulfilling prophecy and move self-perceptions in the direction of being consistent with the stereotype. Also, it is possible that widely held cultural beliefs become prescriptive and thus lead individuals to describe their own behaviour as being in accordance with the stereotype. Gallois (1994), in a review of emotion communication in interpersonal situations, concludes that emotion communication in such situations is highly rule-governed. A similar perspective underlies Averill's (1997) argument regarding the notion of emotion roles that have entrance requirements such that, for example, a successful display of anger presupposes sufficient power to adequately address the anger eliciting issue. Also, Stoppard and Gruchy (1993) found that individuals anticipate costs and rewards for showing certain emotions in certain contexts. Specifically, women expect more costs when not expressing positive emotions in an "other"oriented context, whereas men expect rewards for showing positive emotions regardless of context.

Taken together, these findings from diverse domains suggest that selfperceptions of emotional reactions should show a clear overlap with stereotypical expectations within any particular culture. To the degree that we assume that these influences are due to recollection biases, we may further speculate that the overlap is less pronounced when asking about behavioural reactions in particular social contexts than when asking for reactions in "general" or independent of a specific social situation. Specifically, as data on actual behaviour suggest relatively few sex differences in emotional behaviour whereas the stereotype suggests a wide range of such differences, differences in self-perceptions are more likely to be due to the influence of the cultural stereotypes than to actual behavioural differences between the genders. Study 2 is focused on an analysis of this possibility.

\section{STUDY 2}

Study 1 confirmed that, at least in the Quebec culture, there are widely shared beliefs concerning how men and women differ in their emotion-relevant expressive behaviour. The central issue to be addressed in Study 2, therefore, is the degree of parallelism that exists between perceptions of how typical males and females are perceived to respond to emotional events (Study 1) and selfperceptions of one's own emotional expressivity.

To answer this question, men and women, drawn from the same population as in Study 1, were asked to indicate for each of the seven types of emotion situations employed in Study 1 to what degree they expected to react themselves with each of nine emotions if this event should happen to them. In addition, we asked participants to indicate the likelihood that they would show each of nine types of behaviours corresponding to the nine emotions of the emotion profile. These measures were embedded in a larger questionnaire that assessed beliefs 
regarding emotional reactions of other social groups. ${ }^{4}$ This placement made sure that the cultural beliefs and stereotypes held by the participants regarding emotional expressivity in general were evoked. However, the specific issue of sex differences was not alluded to.

\section{Method}

Participants. A total of 360 students attending junior colleges in the Montreal urban region participated in the study. Of these, only those participants whose first language learned and still spoken was French, who were born in the province of Quebec (Canada), who had not lived outside of Quebec for more than five consecutive years, and who identified themselves as either Canadian or Quebecois and not as a member of any other group were retained. ${ }^{5}$ The resulting sample consisted of 289 individuals. In total, 163 women and 126 men with a mean age of 18.86 years participated in groups of between 15 and 40 participants. Of these, 63 men and 85 women completed the emotion profiles and 63 men and 78 women completed the behavioural reactions scale.

Procedure. Participants were informed of their right to discontinue participation at any time and for any reason. The experimenter then distributed the questionnaires. The instructions as well as an example were provided on the first page. The same instructions as employed in Study 1 were used, the only difference being that participants were told to think about how they would react and to indicate the likelihood in percent that they themselves would react with each of the emotions/behaviours indicated. The same instructions were given for both the emotion profile and the behavioural reactions scale with the only difference being that for the latter the word "emotion", was replaced with "behaviour".

Materials. For each of seven emotions (happiness, anger, fear, sadness, disgust, shame, and guilt) two of the four vignettes employed in Study 1 were retained. Each participant responded to only one vignette for each emotion and vignettes were counterbalanced across participants.

\footnotetext{
${ }^{4}$ In the present context only data from the part of the questionnaire asking participants about how they themselves would react in the described situation will be presented.

${ }^{5}$ These selection criteria were included because the participants for Study 2 were recruited from several Montreal junior colleges with an ethnically diverse population. Emotion stereotypes are well known to differ between cultures (Kirouac \& Hess, 1999) and the participants in Study 1 were recruited from an ethnically homogenous population. To ensure comparability between the two samples, it was therefore necessary to exclude participants from groups not present in the first sample.
} 


\section{Dependent measures.}

Emotion profiles. These were computed in the same manner as in Study 1. That is, responses were averaged across the vignettes representing each specific emotion theme.

Behavioural reactions scale. This scale is intended to assess the behavioural reactions that are expected for someone who is experiencing the emotional state in question. To construct the scale, a two-step procedure was employed.

First, 68 students of the University of Quebec at Montreal, 54 women and 14 men, with a mean age of 27.9 years, were asked to list for each of nine emotions (happiness, serenity, anger, disgust, fear, contempt, sadness, shame, and guilt) the behaviours and observable reactions they associate with each specific emotion. The answers were compiled into frequency lists and synonyms were grouped together. The three most frequently named behaviours for each emotion were retained. Due to overlap between some emotions this procedure yielded a list of 22 behaviours. For the second step, a total of 44 participants, 28 women and 16 men with a mean age of 22.6 years, were asked to rate for each behaviour and each of the nine emotions the likelihood of observing the behaviour in a person experiencing the emotion using a 7-point scale (1, very unlikely; 7, very likely). Behaviours that received a mean rating of 6 or above for only one emotion comprise the final scale.

These consisted of the following items: ${ }^{6}$ to laugh/to get excited (happiness), to tremble/to freeze (fear), to hit/to insult/to criticise (anger), to cry/to isolate oneself (sadness), to grimace/to vomit (disgust), to stare/to look hard at (contempt), to smile/to relax/to contemplate (serenity), to withdraw/to lower one's head/to blush (shame), to justify oneself/to apologise (guilt). On the profile measure, participants were asked to indicate the likelihood, as a percentage, that they would show each of the behaviours in a situation like the one described in the vignettes.

Data analyses. Each participant responded to one scenario for each of the seven emotions themes and used only one type of scale. As in Study 1, the data were collapsed over scenarios. Because we had responses from more women than men for both dependent measures, the data for the female participants were weighted to create equal cell sizes. Profile analyses across the nine emotional reactions/behaviours were conducted to assess sex differences.

\footnotetext{
${ }^{6}$ These items were translated from the original French by the authors.
} 


\section{Results and discussion}

As noted earlier, the goal of Study 2 was to assess to what degree the selfperceptions of the men and women who participated in this study overlap with the general stereotypes found in Study 1. To accomplish this, the emotion profiles from Study 1 were compared with the self-reported emotional profiles and with the behavioural reaction profiles collected here.

Self-described emotion profiles. The means and standard deviations for the nine scales of the emotion profiles for male and female participants are presented in Table 3. A comparison of these means with those of the profiles from Study 1 reveals substantial similarities between the emotional reactions expected for oneself and those expected for men and women in general.

Behavioral reactions. Table 3 shows the means and standard deviations for the nine behavioural reaction profiles. These profiles represent the participants self-report of the likelihood that they would display these specific behaviours in the situations described by the vignettes. As the focus of this paper is on sex

TABLE 3

Means and standard deviations for self-perceptions of emotional reactions/behaviours for men and women (Study 2)

\begin{tabular}{|c|c|c|c|c|}
\hline \multirow[b]{2}{*}{ Situation } & \multicolumn{2}{|c|}{ Men } & \multicolumn{2}{|c|}{ Women } \\
\hline & Mean & $S D$ & Mean & $S D$ \\
\hline \multicolumn{5}{|l|}{ Anger } \\
\hline Happiness & 2.62 & 12.24 & 0.06 & 0.54 \\
\hline Laugh/get excited & 7.90 & 18.26 & 4.29 & 11.68 \\
\hline Serenity & 2.62 & 8.27 & 1.35 & 5.32 \\
\hline Smile/relax/contemplate & 8.65 & 20.68 & 4.35 & 11.85 \\
\hline Sadness & $23.59 * * *$ & 23.90 & 44.05 & 31.51 \\
\hline Cry/isolate oneself & $9.35^{*}$ & 20.83 & 18.25 & 22.50 \\
\hline Fear & 6.03 & 15.89 & 4.86 & 10.70 \\
\hline Tremble/freeze & 3.32 & 8.14 & 6.30 & 11.55 \\
\hline Anger & 62.68 & 28.73 & 60.76 & 29.28 \\
\hline Hit/insult/criticise & 35.40 & 30.42 & 26.54 & 28.73 \\
\hline Disgust & 11.75 & 24.47 & 10.94 & 19.97 \\
\hline Grimace/vomit & 1.94 & 5.53 & 2.86 & 8.54 \\
\hline Contempt & 18.65 & 25.05 & 21.02 & 25.43 \\
\hline Stare/look hard at & $20.65^{*}$ & 27.18 & 11.56 & 22.16 \\
\hline Shame & $2.86^{*}$ & 8.27 & 8.02 & 18.87 \\
\hline Withdraw/lower one's head/blush & 10.44 & 21.87 & 10.38 & 16.42 \\
\hline Guilt & 3.49 & 8.26 & 4.91 & 15.82 \\
\hline Justify oneself/apologise & 20.45 & 30.72 & 17.61 & 26.63 \\
\hline
\end{tabular}


TABLE 3

(Continued)

\begin{tabular}{|c|c|c|c|c|}
\hline \multirow[b]{2}{*}{ Situation } & \multicolumn{2}{|c|}{ Men } & \multicolumn{2}{|c|}{ Women } \\
\hline & Mean & $S D$ & Mean & $S D$ \\
\hline \multicolumn{5}{|l|}{ Disgust } \\
\hline Happiness & $6.11^{*}$ & 17.14 & 1.43 & 5.75 \\
\hline Laugh/get excited & $19.89^{*}$ & 26.69 & 9.15 & 18.25 \\
\hline Serenity & 6.11 & 18.55 & 2.08 & 11.59 \\
\hline Smile/relax/contemplate & $11.59^{\mathrm{t}}$ & 21.13 & 5.78 & 14.82 \\
\hline Sadness & 5.16 & 15.37 & 4.90 & 15.31 \\
\hline Cry/isolate oneself & 0.73 & 4.03 & 3.46 & 13.52 \\
\hline Fear & $3.73^{\mathrm{t}}$ & 10.66 & 9.11 & 20.18 \\
\hline Tremble/freeze & $4.76^{\mathrm{t}}$ & 11.54 & 10.15 & 19.77 \\
\hline Anger & 13.59 & 21.46 & 14.37 & 25.21 \\
\hline Hit/insult/criticise & 12.14 & 22.10 & 11.86 & 21.51 \\
\hline Disgust & 50.86 & 32.57 & 58.81 & 31.78 \\
\hline Grimace/vomit & 11.67 & 21.21 & 16.92 & 22.36 \\
\hline Contempt & 17.98 & 22.26 & 17.50 & 27.06 \\
\hline Stare/look hard at & 19.91 & 23.62 & 16.86 & 23.63 \\
\hline Shame & 10.23 & 19.72 & 16.83 & 27.38 \\
\hline Withdraw/lower one's head/blush & 6.45 & 16.63 & 11.69 & 23.37 \\
\hline Guilt & 2.62 & 9.37 & 1.19 & 3.78 \\
\hline Justify oneself/apologise & 9.92 & 25.11 & 7.44 & 19.24 \\
\hline \multicolumn{5}{|l|}{ Sadness } \\
\hline Happiness & 3.17 & 12.80 & 4.12 & 15.94 \\
\hline Laugh/get excited & 1.23 & 7.89 & 1.28 & 1.14 \\
\hline Serenity & $8.87^{\mathrm{t}}$ & 20.17 & 3.18 & 14.56 \\
\hline Smile/relax/contemplate & 6.21 & 18.48 & 3.46 & 11.96 \\
\hline Sadness & 76.90 & 28.16 & 82.51 & 22.58 \\
\hline Cry/isolate oneself & 60.08 & 31.31 & 67.36 & 30.59 \\
\hline Fear & $8.49^{t}$ & 18.20 & 15.46 & 26.37 \\
\hline Tremble/freeze & 15.95 & 27.63 & 11.35 & 20.68 \\
\hline Anger & 18.25 & 26.41 & 22.61 & 29.19 \\
\hline Hit/insult/criticise & 5.32 & 13.99 & 6.41 & 15.17 \\
\hline Disgust & $6.51^{\mathrm{t}}$ & 20.15 & 1.76 & 9.40 \\
\hline Grimace/vomit & 7.90 & 20.17 & 3.53 & 13.18 \\
\hline Contempt & 6.59 & 21.25 & 3.32 & 11.21 \\
\hline Stare/look hard at & 0.00 & 0.00 & 0.45 & 3.97 \\
\hline Shame & 2.14 & 10.50 & 1.41 & 7.11 \\
\hline Withdraw/lower one's head/blush & $20.08^{*}$ & 29.16 & 7.24 & 16.80 \\
\hline Guilt & 15.24 & 24.94 & 21.41 & 29.85 \\
\hline Justify oneself/apologise & 3.15 & 10.65 & 3.91 & 14.78 \\
\hline \multicolumn{5}{|l|}{ Fear } \\
\hline Happiness & 3.17 & 14.87 & 0.06 & 0.54 \\
\hline Laugh/get excited & 2.03 & 10.54 & 0.58 & 3.61 \\
\hline Serenity & $5.87 *$ & 16.98 & 0.82 & 3.27 \\
\hline
\end{tabular}

(Continued overleaf) 
TABLE 3

(Continued)

\begin{tabular}{|c|c|c|c|c|}
\hline \multirow[b]{2}{*}{ Situation } & \multicolumn{2}{|c|}{ Men } & \multicolumn{2}{|c|}{ Women } \\
\hline & Mean & $S D$ & Mean & $S D$ \\
\hline Smile/relax/contemplate & $10.48 * *$ & 22.43 & 1.82 & 6.66 \\
\hline Sadness & 23.33 & 30.51 & 29.29 & 35.88 \\
\hline Cry/isolate oneself & $13.06^{* *}$ & 25.36 & 30.22 & 35.82 \\
\hline Fear & $51.11 * * *$ & 32.07 & 75.35 & 27.15 \\
\hline Tremble/freeze & $20.08 *$ & 25.65 & 33.78 & 33.78 \\
\hline Anger & 24.68 & 32.97 & 19.54 & 29.49 \\
\hline Hit/insult/criticise & $12.22 *$ & 20.98 & 4.46 & 14.10 \\
\hline Disgust & 7.87 & 18.08 & 10.06 & 22.06 \\
\hline Grimace/vomit & 4.29 & 12.70 & 3.65 & 11.15 \\
\hline Contempt & 11.92 & 22.16 & 11.41 & 20.78 \\
\hline Stare/look hard at & 6.77 & 18.22 & 3.91 & 14.05 \\
\hline Shame & 1.43 & 4.70 & 2.00 & 6.15 \\
\hline Withdraw/lower one's head/blush & 16.06 & 25.79 & 10.00 & 21.17 \\
\hline Guilt & 7.94 & 21.99 & 3.69 & 9.95 \\
\hline Justify oneself/apologise & 2.82 & 12.63 & 2.76 & 13.19 \\
\hline \multicolumn{5}{|l|}{ Shame } \\
\hline Happiness & 4.52 & 15.96 & 1.44 & 10.39 \\
\hline Laugh/get excited & 18.25 & 29.05 & 13.87 & 19.90 \\
\hline Serenity & 4.44 & 14.92 & 1.82 & 10.28 \\
\hline Smile/relax/contemplate & $12.06^{\mathrm{t}}$ & 23.33 & 6.23 & 12.62 \\
\hline Sadness & $11.51^{*}$ & 21.21 & 20.77 & 23.61 \\
\hline Cry/isolate oneself & 3.34 & 8.77 & 4.26 & 11.07 \\
\hline Fear & 12.46 & 24.31 & 11.35 & 17.00 \\
\hline Tremble/freeze & 7.76 & 15.69 & 8.94 & 14.91 \\
\hline Anger & 12.62 & 22.59 & 14.32 & 24.08 \\
\hline Hit/insult/criticise & 5.65 & 14.78 & 3.14 & 11.40 \\
\hline Disgust & 2.06 & 9.66 & 3.47 & 12.44 \\
\hline Grimace/vomit & 2.02 & 5.90 & 2.31 & 10.86 \\
\hline Contempt & 7.30 & 15.32 & 8.11 & 18.80 \\
\hline Stare/look hard at & 3.47 & 8.33 & 3.04 & 11.60 \\
\hline Shame & $48.19^{*}$ & 35.34 & 59.68 & 29.48 \\
\hline Withdraw/lower one's head/blush & 25.63 & 29.74 & 28.85 & 29.08 \\
\hline Guilt & $30.95 * *$ & 34.42 & 47.88 & 32.82 \\
\hline Justify oneself/apologise & 43.79 & 31.44 & 42.56 & 30.74 \\
\hline \multicolumn{5}{|l|}{ Guilt } \\
\hline Happiness & $10.24 *$ & 24.32 & 3.55 & 11.89 \\
\hline Laugh/get excited & 7.34 & 22.59 & 5.84 & 17.61 \\
\hline Serenity & 2.62 & 10.08 & 2.23 & 7.71 \\
\hline Smile/relax/contemplate & 6.45 & 16.92 & 4.16 & 14.09 \\
\hline Sadness & $20.03^{\mathrm{t}}$ & 27.05 & 29.71 & 33.90 \\
\hline Cry/isolate oneself & $9.60 * *$ & 19.92 & 22.73 & 29.79 \\
\hline Fear & 17.78 & 26.67 & 26.53 & 33.05 \\
\hline
\end{tabular}


TABLE 3

(Continued)

\begin{tabular}{|c|c|c|c|c|}
\hline \multirow[b]{2}{*}{ Situation } & \multicolumn{2}{|c|}{ Men } & \multicolumn{2}{|c|}{ Women } \\
\hline & Mean & $S D$ & Mean & $S D$ \\
\hline Tremble/freeze & 12.06 & 22.12 & 12.27 & 20.24 \\
\hline Anger & 17.62 & 29.80 & 19.26 & 29.50 \\
\hline Hit/insult/criticise & 1.29 & 5.43 & 2.21 & 7.20 \\
\hline Disgust & 12.48 & 25.69 & 11.52 & 24.02 \\
\hline Grimace/vomit & 5.81 & 16.42 & 3.51 & 13.85 \\
\hline Contempt & 16.03 & 28.57 & 14.64 & 26.11 \\
\hline Stare/look hard at & 1.29 & 4.95 & 1.36 & 4.85 \\
\hline Shame & $26.67 * *$ & 32.27 & 53.75 & 34.67 \\
\hline Withdraw/lower one's head/blush & 19.52 & 24.72 & 17.69 & 23.33 \\
\hline Guilt & 50.71 & 38.85 & 57.53 & 33.72 \\
\hline Justify oneself/apologise & 44.84 & 35.81 & 42.86 & 36.08 \\
\hline \multicolumn{5}{|l|}{ Happiness } \\
\hline Happiness & 89.13 & 19.81 & 91.06 & 14.71 \\
\hline Laugh/get excited & 57.63 & 34.20 & 60.69 & 32.83 \\
\hline Serenity & $27.38^{\mathrm{t}}$ & 36.50 & 39.06 & 37.97 \\
\hline Smile/relax/contemplate & 54.51 & 34.45 & 46.47 & 34.32 \\
\hline Sadness & 1.27 & 9.46 & 0.20 & 1.23 \\
\hline Cry/isolate oneself & 0.40 & 3.18 & 0.29 & 1.41 \\
\hline Fear & 1.03 & 3.72 & 2.06 & 6.80 \\
\hline Tremble/freeze & $4.19^{t}$ & 13.13 & 0.90 & 0.57 \\
\hline Anger & 0.32 & 2.52 & 0.00 & 0.00 \\
\hline Hit/insult/criticise & 0.00 & 0.00 & 0.13 & 1.14 \\
\hline Disgust & 0.48 & 3.78 & 0.00 & 0.00 \\
\hline Grimace/vomit & 0.00 & 0.00 & 0.00 & 0.00 \\
\hline Contempt & 0.81 & 5.22 & 0.00 & 0.00 \\
\hline Stare/look hard at & 3.55 & 13.92 & 1.47 & 3.88 \\
\hline Shame & 1.11 & 7.64 & 0.00 & 0.00 \\
\hline Withdraw/lower one's head/blush & 7.18 & 19.05 & 6.87 & 18.59 \\
\hline Guilt & 2.70 & 15.26 & 0.00 & 0.00 \\
\hline Justify oneself/apologise & 3.55 & 15.35 & 0.90 & 3.40 \\
\hline
\end{tabular}

Note: Significant differences between men and women: ${ }^{\mathrm{t}} p<.1 ;{ }^{*} p<.05 ; * * p<.01 ; * * * p<.001$.

differences, we will not discuss these data in detail. However, two things are noteworthy. First, both men and women report lower probabilities that they would react with a certain behaviour, for example, "to laugh/to get excited", than with the emotion that corresponds to the behaviour (happiness). This finding is not unexpected as individuals do not always express their emotions outwardly or may have developed idiosyncratic means of expressing certain emotions. Second, the profile data for the behaviours resemble strongly the emotional reactions reported. For example, for anger the highest means are found for to hit/to insult/to criticise, for to cry/to isolate one self, and for to stare/ 
to look hard at, which correspond to anger, sadness, and contempt reactions, respectively.

However, a number of differences between these two types of profiles can also be observed. For example, for the emotional reaction profiles for anger and sadness the second highest mean is found for guilt whereas for the behavioural reaction profiles for these emotions the second highest mean is found for shame behaviours (to withdraw/to lower one's head/to blush). This findings may be due to the general confusion between shame and guilt that has been observed for Western cultures (see e.g., Wallbott \& Scherer, 1994).

Sex differences. To assess whether male and female participants differed with regard to their expected emotional reactions and behaviours in the different emotion eliciting situations, profile analyses were conducted on the ratings for each emotion situation. All analyses and post-hoc tests were conducted at an alpha level of .05. The results are shown in Table 4.

TABLE 4

Results of profile analyses on self-reported likelihood of emotional reactions/behaviours for men and women (Study 2)

\begin{tabular}{lccc}
\hline Vignettes & $F$ & $d f$ & $p$ \\
\hline Anger & & & \\
$\quad$ Reactions & 2.95 & 8,116 & .005 \\
$\quad$ Behaviours & 1.83 & 8,113 & .079 \\
Disgust & & & \\
$\quad$ Reactions & 1.69 & 8,115 & .107 \\
$\quad$ Behaviours & 1.73 & 8,116 & .099 \\
Fear & & & \\
$\quad$ Reactions & 3.30 & 8,116 & .002 \\
$\quad$ Behaviours & 3.90 & 8,116 & .001 \\
Sadness & & & \\
$\quad$ Reactions & 2.07 & 8,116 & .044 \\
Behaviours & 1.70 & 8,115 & .105 \\
Guilt & & & \\
$\quad$ Reactions & 1.86 & 8,117 & .071 \\
Behaviours & 1.30 & 8,115 & .251 \\
Shame & & & .376 \\
$\quad$ Reactions & 2.15 & 8,116 & .037 \\
Behaviours & .70 & 8,116 & .689 \\
Happiness & & & \\
Reactions & 1.06 & 8,116 & \\
Behaviours & 1.09 & 8,116 & \\
\hline
\end{tabular}


Emotional reactions. For all emotion situations except disgust and happiness the emotional reactions profiles were nonparallel or marginally nonparallel (see Table 4). For the nonparallel profiles post-hoc tests revealed that a similar pattern of sex differences emerged across the different emotion themes. For happiness and serenity there were either no significant differences between men and women, or men reported experiencing these emotions with a higher probability than did women. Conversely, for sadness, fear, shame, and guilt there were either no significant sex differences or women reported feeling these emotions with a higher probability than did men. For anger, disgust, and contempt reactions no significant differences emerged.

Emotional behaviour profiles. Only the fear profile was significantly nonparallel (different) for men and women. The profiles for anger and disgust were marginally nonparallel and the remaining profiles were all parallel and coincident. Thus, fewer significant differences between men's and women's expected behaviours in the situations described by the vignettes were found than was the case for the expected emotional reactions in Studies 1 and 2. Yet, it is important to note that the probabilities reported for the behavioural profiles were lower overall and it is thus possible that this lack of differences is due to a lack of sensitivity of the measure.

As postulated based on previous research (e.g., Crawford, Clippax, Onyx, Gault, \& Benton, 1992) as well as on the emotional reactions profile, women report a higher probability of either crying or isolating oneself in an anger situation than do men. Conversely, and in accordance with both the literature on anger expression (see earlier) and the results from the emotional reactions profile, men report it more likely to hit/to insult/to criticise than women, though this difference is not significant. In addition, they report that they are significantly more likely to stare/to look hard (contempt) than do women, a behaviour that could also be interpreted as aggressive when shown in response to an insult.

As regards the disgust situations, men report that they are more likely to laugh/to get excited. This sex difference matches the finding for the emotional reactions profile but was not found in Study 1 . Finally for the fear situation, men report that they are more likely to hit/to insult/to criticise as well as to smile/to relax/to contemplate (serenity) than do women. The emotional reactions profile for fear from this study shows that men report a higher probability of reacting with serenity, but not with anger, than do women. As these behaviours correspond to a macho or cool demeanour in a threatening situation they fit the general stereotype of masculine behaviour. Conversely, women report to be more likely to tremble/to freeze as well as to cry/to isolate one self in fear situations.

In sum, although many fewer sex differences reached significance when comparing self-reported expected behaviours for men and women, the pattern of 
results is largely congruent with previous findings. Specifically, women reported a higher probability of showing behaviours congruent with sadness and fear than did men, whereas men reported higher probabilities for anger and serene behaviours. In addition, men reported a higher probability of showing a contempt behaviour in an anger situation, yet the relevant behaviour (to stare/to look hard) could be interpreted as aggressive in that context.

\section{How congruent are the findings from Studies 1 and 2?}

As mentioned earlier, not all significant differences found in Study 1 for the expected reactions for men and women in general, were also found for selfreports of expected reactions by men and women for themselves. Yet, as the results for the two studies are based on different degrees of freedom, significance levels cannot be directly compared. To assess whether stereotypical and self-reported sex differences were of similar magnitude, we computed the effect sizes for both studies. Table 5 shows Cohen's $d$ for the post-hoc

TABLE 5

Cohen's $d$ for sex differences in Studies 1 and 2

\begin{tabular}{|c|c|c|c|}
\hline \multirow[b]{2}{*}{ Situation } & \multirow{2}{*}{$\begin{array}{c}\text { Study } 1 \\
\text { Emotional } \\
\text { reactions }\end{array}$} & \multicolumn{2}{|c|}{ Study 2} \\
\hline & & $\begin{array}{l}\text { Emotional } \\
\text { reactions }\end{array}$ & $\begin{array}{l}\text { Emotional } \\
\text { behaviours }\end{array}$ \\
\hline \multicolumn{4}{|l|}{ Anger } \\
\hline Happiness & & & -0.23 \\
\hline Serenity & & & -0.25 \\
\hline Sadness & 2.03 & 0.73 & 0.41 \\
\hline Fear & 1.23 & & 0.30 \\
\hline Anger & -0.55 & & -0.30 \\
\hline Disgust & 0.68 & & \\
\hline Contempt & & & -0.26 \\
\hline Shame & 1.17 & -0.35 & \\
\hline Guilt & 1.29 & & \\
\hline \multicolumn{4}{|l|}{ Disgust } \\
\hline Happiness & -0.32 & -0.37 & -0.47 \\
\hline Serenity & -0.45 & -0.26 & -0.21 \\
\hline Sadness & 0.95 & & 0.27 \\
\hline Fear & 1.89 & 0.33 & 0.33 \\
\hline Anger & & & \\
\hline Disgust & 1.60 & 0.24 & 0.24 \\
\hline Contempt & 0.50 & & \\
\hline Shame & 0.62 & -0.28 & 0.26 \\
\hline Guilt & 0.62 & 0.20 & \\
\hline
\end{tabular}


TABLE 5

(Continued)

\begin{tabular}{|c|c|c|c|}
\hline \multirow[b]{2}{*}{ Situation } & \multirow{2}{*}{$\begin{array}{c}\text { Study } 1 \\
\begin{array}{c}\text { Emotional } \\
\text { reactions }\end{array}\end{array}$} & \multicolumn{2}{|c|}{ Study 2} \\
\hline & & $\begin{array}{c}\text { Emotional } \\
\text { reactions }\end{array}$ & $\begin{array}{l}\text { Emotional } \\
\text { behaviours }\end{array}$ \\
\hline \multicolumn{4}{|l|}{ Sadness } \\
\hline Happiness & -0.53 & & \\
\hline Serenity & & -0.32 & \\
\hline Sadness & 1.84 & 0.22 & 0.24 \\
\hline Fear & 1.71 & 0.31 & \\
\hline Anger & -0.65 & & 0.42 \\
\hline Disgust & & -0.30 & -0.25 \\
\hline Contempt & 0.21 & & \\
\hline Shame & 0.20 & -0.46 & 0.54 \\
\hline Guilt & 1.76 & 0.22 & -0.33 \\
\hline \multicolumn{4}{|l|}{ Fear } \\
\hline Happiness & -0.21 & -0.30 & \\
\hline Serenity & -0.26 & -0.41 & -0.52 \\
\hline Sadness & 1.89 & & 0.55 \\
\hline Fear & 2.21 & 0.82 & 0.46 \\
\hline Anger & -0.84 & & -0.43 \\
\hline Disgust & 0.67 & & \\
\hline Contempt & & & \\
\hline Shame & 0.49 & & -0.26 \\
\hline Guilt & 1.72 & -0.25 & \\
\hline \multicolumn{4}{|l|}{ Shame } \\
\hline Happiness & -0.45 & -0.23 & \\
\hline Serenity & -0.27 & -0.20 & -0.31 \\
\hline Sadness & 1.75 & 0.41 & \\
\hline Fear & 1.28 & & \\
\hline Anger & -0.69 & & \\
\hline Disgust & 0.65 & & \\
\hline Contempt & 0.54 & & \\
\hline Shame & 1.54 & 0.35 & \\
\hline Guilt & 1.53 & 0.50 & \\
\hline \multicolumn{4}{|l|}{ Guilt } \\
\hline Happiness & -1.07 & -0.35 & \\
\hline Serenity & -0.51 & & \\
\hline Sadness & 1.98 & 0.32 & 0.51 \\
\hline Fear & 1.62 & 0.29 & \\
\hline Anger & & & \\
\hline Disgust & 1.28 & & \\
\hline Contempt & 0.75 & & \\
\hline Shame & 1.78 & 0.51 & \\
\hline Guilt & 1.83 & 0.20 & \\
\hline
\end{tabular}


comparisons for each emotion in the profile for the six negative emotion situations across the two studies. For Study 1 the effect sizes for the urban and the rural sample were combined. To facilitate the reading of the table only differences for which $d$ was at least 0.20 (i.e., equivalent to a small effect) are shown. In general, effect sizes for Study 1 are larger than those for Study 2. For emotional reactions the expected differences between men and women were of medium $(>0.50)$ to large $(>0.80)$ size, whereas for self-perceptions the expected differences between men and women were small to medium in size.

The most stable sex difference across the two studies was found for sadness, both as a core emotion in sadness vignettes and as a secondary emotion. That is, for all negative emotions the general stereotype describes women as reacting with more sadness, and women also perceive themselves as reacting with more sadness and/or report more sadness behaviours. Further, women are expected to react with (and they self-report) more shame and guilt reactions/behaviours in shame and guilt situations. However, for the other negative emotions (anger, disgust, sadness, and fear) men report higher levels of shame reactions or shame behaviours (but not both). Finally, fear reactions and behaviours are described as more likely for women in general and women perceive themselves as reacting with more fear in disgust, sadness, fear, and guilt situations.

Another very stable sex difference across studies regards happiness and serenity reactions for negative emotion vignettes. Men consistently are both expected to react with and self-report more happiness reactions as well as happiness behaviours for disgust, fear, and shame vignettes. To a lesser degree this pattern also emerges for sadness and guilt vignettes. As mentioned in the discussion of Study 1, this may reflect differences in the value structure between men and women.

In sum, a comparison of effect sizes shows considerable consistency across the two studies. Specifically noteworthy is the very consistent finding for both the stereotypical beliefs and the self-reports with regard to sadness reactions, which are considered as more likely for women than for men for all negative emotion situations studied.

However, a number of differences between the general stereotypes and the self-perceptions also emerged, specifically with regard to shame reactions. Whereas women are generally believed to be more likely to react with shame to negative emotion events, the self-reported likelihood for shame as a concomitant emotion is higher for men for all situations except for guilt (given the general confusion regarding these states observed in Western cultures, this may be due to shame being a core rather than a concomitant emotion for guilt scenarios). Yet, self-reported shame behaviours are more likely for women than for men in sadness and disgust situations. A possible explanation is that men are actively trying to not show shame reactions-to conform to the general stereotype-but are aware that they nonetheless are likely to feel shame in those situations. 
To conclude, the general stereotype and self-perceptions of emotional reactions and behaviours suggest that women react with more sadness to all types of negative emotion events. Further, the stereotypes and the self-perceptions are in agreement on men's greater likelihood to react with happiness and serenity to a variety of negative emotions. In contrast, differences between the general stereotypes and self-perceptions emerge for shame reactions with men reporting, in situations when shame is a secondary emotion, a higher likelihood of shame reactions, but not necessarily shame behaviours, than do women.

\section{STUDY 3}

The results from Study 2 suggest that the stereotypical beliefs regarding men's and women's emotional reactions and behaviours in a variety of emotioninducing situations converge with self-perceptions of men's and women's emotional reactions and behaviours in those same situations. This finding is consistent with the notion that autobiographic statements that require participants to summarise over a number of related experiences may be biased by stereotypical expectations (see e.g., Feldman-Barrett et al., 1998). This leads to the question of whether a similar overlap can also be found for narratives of single, specific emotional events from the participants' past. If stereotypes indeed influence the way emotional events are reconstructed in a narrative, we would expect to find that women report more sadness events and express more sadness overall as well as more intense target emotions for all but anger events. Conversely, we would expect men to report more situations involving anger as well as experiencing more happiness and serenity overall.

However, as Study 3 is based on personal narratives and not on standardised vignettes, such a finding could simply be due to the fact that the emotion antecedent events in a real-life setting differ for men and women. To assess this possibility we decided to measure the emotion antecedent events by means of an appraisal questionnaire that assesses the participants' perception of the emotion antecedent situation. To the degree that perceptions of emotion antecedents are similar for men and women while reported emotional reactions differ we can conclude that observed sex differences are not due to differences in the perception of the event per se. For example, if women's tendency to report more sadness were due to a difference in the perception of the emotion antecedent situation, we would expect women to report more often that they did not feel that they could do anything to change the situation or that they had to adapt to the situation (as sadness is generally associated with the notion of irrevocable loss).

\section{Overview}

For this study we translated and adapted a questionnaire reported by Scherer (1988) that consists of two parts. The first part invites the participants to describe an event during which they experienced an emotion. The second part 
asks specific questions regarding the appraisal process. We added a section in which we asked participants to complete an emotion profile for the event. To avoid the possibility that men and women would relate narratives from widely different types of situations we asked participants to describe an event related to family interactions. This class of situations should be readily accessible to all participants and ensure that emotional events involving human interaction partners were reported.

Allowing participants to choose the type of emotional events they report has several advantages. Most importantly, we can assess what type of emotional events men and women choose to report spontaneously. It also permits participants to choose a salient event for which they can recollect the information necessary to respond to the appraisal questions. On the other hand, this approach is unlikely to result in a well-balanced distribution of emotion topics, thus making it difficult or impossible to analyse the data separately for different emotion themes. Yet, in the context of the present study we were only interested in sex differences in reports and not in absolute levels of responses. Studies 1 and 2 suggest that sex differences are very stable across all negative emotions. We therefore decided to allow participants to choose the emotion theme and analysed the data collapsed across all negative emotion themes.

\section{Method}

Participants. A total of 171 students, 87 men and 84 women with a mean age of 18.62 years, participated in this study. Most of the participants (147) were recruited from an ethnically homogenous junior college in the larger Montreal urban area (Cégep Lionel-Groulx, Ste-Thérèse, Québec, Canada) and participated in groups. An additional 24 participants were recruited individually.

Procedure. The experimenter explained that the study concerned emotional events in people's lives. Participants were informed of their right to discontinue participation at any time and for any reason. The experimenter then distributed the questionnaires and responded to questions regarding the procedure. The task took between 10 and 30 minutes. Most of this time difference can be attributed to the amount of detail provided in the event description; that ranged from a couple of words (e.g., birth of my daughter) to well over a page.

Dependent measures. The questionnaire consisted of four parts. The first part was an open-ended question asking the participants to describe any event involving their family life that led them to experience an emotion. Sadness, shame, anger, guilt, joy, fear, and disgust were provided as examples of emotions. Participants were asked to give a detailed description of the situation including the events that led up to it, their reactions and those of others present. In addition, they were asked to identify the people involved with respect to their 
relationship with them (e.g., my partner, my sister, etc.) as well as to identify the main emotion theme of the story. This served to ensure that the participants based their answers to the following questions on a specific event and not on a class of similar events.

Emotion description. The second part of the questionnaire consisted of questions regarding when the event happened, for how long they felt the emotion, and the intensity of the core emotion identified as the emotion theme of the story. Participants were also asked to indicate whether they tried to hide or control their feelings and to what degree. This part also contained the emotion profile used in Studies 1 and 2. Using this measure, participants were asked to indicate to what percentage they had felt each of the nine emotions from the profile.

Appraisal questionnaire. A series of questions regarding the appraisal process were used to assess the emotion antecedent events. These questions were translated from the questionnaire reported by Scherer (1988). Specifically, participants were asked to what degree they had expected the situation to occur, how pleasant or unpleasant they considered the event, whether the event helped or hindered them in achieving their aims, to what degree they considered that the situation or the event that caused the emotion was unjust or unfair, whether the event was caused by their own or by someone else's behaviour, to what degree they considered that the behaviour that caused the event would be judged as improper or immoral by their acquaintances, how the event affected their feelings about themselves such as their self-esteem or self-confidence, and finally to what degree the event changed their relationships with the people involved (all on negative-positive 7-point bipolar scales).

In addition, participants were given a list of 11 specific potential causes of the event (caused it myself, natural causes, act of god, etc.). They also evaluated their ability to act upon or to cope with the event and its consequences using a scale involving 5 options. The last part of the questionnaire consisted of questions regarding the participants' sex, their age, and whether they were currently seeing someone in a love-based relationship and if so for how long.

\section{Results}

Of the 171 reported emotion events, 147 ( 74 reported by men and 73 reported by women) concerned negative emotional events. Of these, a total of 62 were sadness events, 22 were anger events, and 11 were guilt events. The remaining events were distributed over a large number of negative emotions.

As expected, the very low frequency of reports for specific emotion themes made separate analyses of reports of these events unfeasible. We therefore decided to perform analyses on the data summed over all negative emotion themes. The 24 events with positive emotion themes were obviously excluded from these analyses. 
Do women report more sadness events and men more anger events? To address this question a chi-square test was performed on the frequency with which men and women labeled the reported event as a sad versus an anger event. In line with the results of Studies 1 and 2, we found men to report more anger events and women to report more sadness events. Specifically, 14 men and 8 women reported anger events and 25 men and 37 women reported sad events, $\chi^{2}$ $=3.55, p=.06, d=.42$.

Do women report more intense core emotions than men? For the intensity of the core emotion experienced during the event women reported significantly higher levels $(\mathrm{M}=5.93, \mathrm{SD}=1.01)$ than men $(\mathrm{M}=5.54, \mathrm{SD}=1.23), t(145)=$ 2.22, $p=.028, d=.37$.

Do women and men report different levels of negative emotions across the emotion profile? Figure 2 presents the emotion profiles separately for men and women. An inspection of the profiles shows that overall men report more happiness/serenity whereas women report higher levels of all other emotions. The profiles are nonparallel, $F(8137)=58.77, p<.001$. Post-hoc analyses revealed a significant sex difference in self-reported sadness with women reporting higher levels than men. To compare the sex differences in the profiles

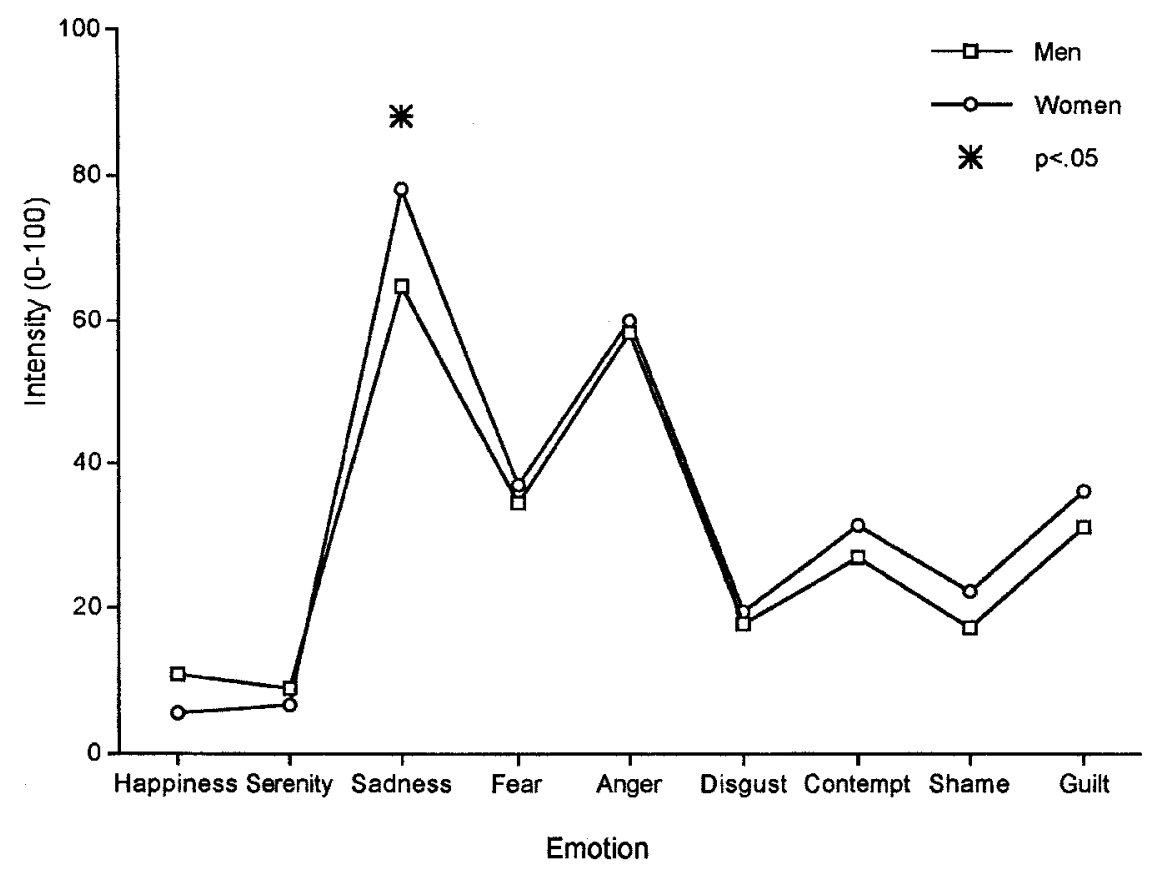

Figure 2. Emotion profile for men's and women's self-reported emotional reactions to negative emotion events (Study 3). 
found in this study with those from Studies 1 and 2, effect sizes were calculated. Using the same cut-off point as previously (small effect size $d=0.20$ ), only two differences were found to be stable across the three studies. Men report higher levels of happiness for negative emotion events $(d=-0.22)$ and women report higher levels of sadness $(d=0.43)$. As the latter difference may be due to the higher frequency of reported sadness events by women, we also verified whether women report higher levels of sadness when sadness events are excluded from the analysis. An inspection of the means revealed a marginally significant difference in the same direction: $M=64.72, \mathrm{SD}=32.47$ and $M=51.08, \mathrm{SD}=$ 36.84 , for women and men respectively, $t(83)=1.81, p=.074, d=.40$.

Are there differences in appraisal patterns between men and women? As mentioned earlier, the reported differences in emotional reactions could be due to differences in the perception of the emotion antecedent events. Yet, no differences in appraisal patterns were found, suggesting that emotion antecedent events were perceived similarly. In addition, despite the more frequent and more intense reports of sadness, women did not report different coping styles than men and did not seem to feel more helpless (38 men vs. 34 women report this reaction). In fact, women more frequently report that they felt able to "influence the situation positively" (19 women vs. 9 men report this reaction, though the difference is not significant).

\section{Discussion}

Study 3 extended the study of emotion stereotypes and self-perceptions based on vignettes to narratives of emotional events that the participants had in fact experienced themselves. The goal of the study was to investigate whether the findings from Studies 1 and 2 generalise to such recollections of personal events. The results strongly suggest this to be the case. Women again report higher levels of sadness for negative emotion events. They also report more sad events in general. Men on the other hand, report more anger events as well as more happiness for negative emotion events as was found in both Studies 1 and 2. Yet, no reported differences in appraisal or coping emerged. This suggests that the observed differences in emotional reactions are not due to a bias in the appraisal process nor to an inability to act upon the event.

If emotion antecedents do not differ why are differences in emotional reactions reported? We suggest that such differences represent an influence of the general emotion stereotype on the narrative reconstruction of a personal event. This conclusion is based on the observation of a null effect and thus open to the critique that the present study lacked the power necessary to detect real differences in appraisal between men and women. To assess this critique we calculated the absolute mean effect size for the interval-scaled appraisal questions to be $d=0.15$. To achieve $80 \%$ power a sample of 1100 participants would be needed. These data underscore the very slim differences in appraisals 
between men and women, making it highly unlikely that the effects observed in this study are mediated by a difference in appraisal and thus by a difference in the perception of the underlying event.

\section{CONCLUSIONS}

The present series of studies had the goal to assess general emotion stereotypes and their influence on self-perceptions of emotional reactions by men and women. We wanted to both describe these stereotypes in more detail with regard not only to the modal emotion for a specific event (such as sadness reactions to a loss) but also with regard to the secondary emotions present in these situations. Further, we wanted to assess to what degree such expectations for men and women in general, are endorsed by individuals for themselves as well as whether they would be spontaneously reported when relating a personally experienced event.

Two gender differences were found to be stable across all three experimental paradigms. Women are expected to be more likely to react with sadness in all negative emotion situations, they expect themselves to be more likely to react with sadness as well as to cry and to withdraw more, and finally women report more sadness in personal events. In contrast, men are expected to react with more happiness/serenity during negative emotional situations, they expect themselves to react more frequently this way as well as to laugh and smile more and to be more relaxed. They also tend to report more happiness when describing negative personal events.

Not all general emotion stereotypes were found in the self-perceptions studied in Studies 2 and 3. Specifically, according to Study 1 women are expected to be more likely to react with shame and guilt in all negative emotion situations. Yet, women did not expect to do so for themselves in situations other than shame and guilt situations and, in fact, men reported to be more likely to react with shame in a variety of situations. Further, men and women did not report any differences with regard to experiences of shame and guilt during the personal events.

Study 3 included an appraisal questionnaire to address the question whether the self-reported differences in emotionality between men and women are due to differences in the appraisal of the emotion antecedent events or rather to the influence of the prevalent norms. No differences in appraisals were detected suggesting that the self-reported differences may be due to the normative forces of the general stereotype rather than to appraisal differences.

It may be argued that the Study 3 is based on recollections of past events and that self-reports of emotional experiences represent after the fact reconstructions that differ from the actually experienced emotions during the event (see Parkinson \& Manstead, 1993). A related argument may be made for Study 2 where participants had to estimate the likelihood of their emotional reactions to 
hypothetical events (even though the latter were chosen to be common everyday events). This may well be true. Yet, the question remains why did men and women reconstruct the events differently in terms of their emotional reactions? The answer would then be that the prevalent emotion stereotype, which is largely endorsed by the population studied, influenced this reconstruction. Yet, as peoples' sense of self depends on their past histories this would imply, that reconstructions of emotional events in line with emotion stereotypes may eventually lead individuals to conform more closely to these stereotypes as they become more and more part of their emotional history and eventually of their emotional self.

Manuscript received 30 May 1997 Revised manuscript received 23 February 2000

\section{REFERENCES}

Adams, S., Kuebli, J., Boyle, P.A., \& Fivush, R. (1995). Gender differences in parent-child conversations about past emotions: A longitudinal investigation. Sex Roles, 33, 309-323.

Allen, J.G., \& Haccoun, D.M. (1976). Sex differences in emotionality: A multidimensional approach. Human Relations, 29, 711-722.

Averill, J.R. (1997). The emotions: An integrative approach. In R. Hogan, J.A. Johnson, \& S.R. Briggs (Eds.), Handbook of personality psychology (pp. 513-541). San Diego, CA: Academic Press.

Birnbaum, D.W. (1983). Preschoolers' stereotypes about sex differences in emotionality: A reaffirmation. Journal of Genetic Psychology, 143, 139-140.

Briton, N.J., \& Hall, J.A. (1995). Gender-based expectancies and observer judgements of smiling. Journal of Nonverbal Behavior, 19, 49-65.

Brody, L.R., \& Hall, J.A. (1993). Gender and emotion. In M. Lewis \& J.M. Haviland (Eds.), Handbook of emotions (pp. 447-460). New York: Guilford Press.

Crawford, J., Clippax, S., Onyx, J., Gault, U., \& Benton, P. (1992). Emotion and gender: Constructing meaning from memory. London: Sage.

Dunn, J., Bretherton, I., \& Munn, P. (1987). Conversations about feeling states between mothers and their children. Developmental Psychology, 23, 132-139.

Eagly, A.H., \& Mladinic, A. (1989). Gender stereotypes and attitudes toward women and men. Personality and Social Psychology Bulletin, 15, 543-558.

Ekman, P., \& Friesen, W. (1986). A new pan-cultural facial expression of emotion. Motivation and Emotion, 10, 159-168.

Feldman-Barrett, L., Robin, L., Pietromonaco, P.R., \& Eysell, K.M. (1998). Are women the more emotional sex? Evidence from emotional experiences in social context. Cognition and Emotion, $12,555-578$.

Fernandez-Dols, J.-M., \& Ruiz-Belda, M.-A. (1995). Are smiles a sign of happiness? Gold medal winners at the Olympic Games. Journal of Personality and Social Psychology, 69, 1113-1119.

Fischer, A. (1993). Sex differences in emotionality: Fact or stereotype? Feminism and Psychology, 3 , 303-318.

Fivush, R. (1989). Exploring differences in the emotional content of mother child conversation s about the past. Sex Roles, 20, 675-691.

Frank, M.G., \& Ekman, P. (1993). Not all smiles are created equal: The differences between enjoyment and nonenjoyment smiles. [Special Issue: Current issues in psychological humor research.] Humor: International Journal of Humor Research, 6, 9-26. 
Gallois, C. (1994). Group membership, social rules, and power: A social psychological perspective on emotional communication. Journal of Pragmatics, 22, 301-324.

Hall, J.A. (1984). Nonverbal sex differences: Communication accuracy and expressive style. Baltimore, MD: Johns Hopkins University Press.

Izard, C. (1972). Patterns of emotions: A new analysis of anxiety and depression. New York: Academic Press.

Jussim, L., Milbourn, M., \& Nelson, W. (1991). Emotional openness: Sex-role stereotypes and selfperceptions. Representative Research in Social Psychology, 19, 35-52.

Kirouac, G., \& Hess, U. (1999). Group membership and the decoding of nonverbal behavior. In P. Philippot, R. Feldman, \& E. Coats (Eds.), The social context of nonverbal behavior (pp. 182210). New York: Cambridge University Press.

Kring, A.M., \& Gordon, A.H. (1998). Sex differences in emotion: Expression, experience and physiology. Journal of Personality and Social Psychology, 74, 686-703.

LaFrance, M., \& Banaji, M. (1992). Toward a reconsideration of the gender-emotion relationship. In M.S. Clark (Ed.), Emotion and social behavior. Review of personality and social psychology (Vol. 14, pp. 178-201). Newbury Park, CA: Sage.

LaFrance, M., \& Hecht, M.A. (1995). Why smiles generate leniency. Personality and Social Psychology Bulletin, 21, 207-214.

Lazarus, R.S. (1991). Emotion and adaptation. Oxford, UK: Oxford University Press.

Parkinson, B., \& Manstead, A.S.R. (1993). Making sense of emotion in stories and social life. Cognition and Emotion, 7, 295-323.

Plutchik, R. (1980). Emotion: A psychoevolutionary synthesis. New York: Harper \& Row.

Rimé, B., Mesquita, B., Philippot, P., \& Boca, S. (1991). Beyond the emotional event: Six studies on the social sharing of emotion. Cognition and Emotion, 5, 435-465.

Scherer, K.R. (1988). Appendix C: Questionnaire used in the International Survey on Emotion Antecedents and Reactions (ISEAR). In K.R. Scherer (Ed.), Facets of emotion: Recent research. Hillsdale, NJ: Erlbaum.

Sherman, J.W., \& Bessenhoff, G.R. (1999). Stereotypes as source monitoring cues: On the interaction between episodic and semantic memory. Psychological Science, 10, 106-110.

Stoppard, J.M., \& Gruchy, C.D. (1993). Gender, context, and expression of positive emotion. Personality and Social Psychology Bulletin, 19, 143-150

Tucker, J.S., \& Riggio, R.E. (1988). The role of social skills in encoding posed and spontaneous facial expressions. Journal of Nonverbal Behavior, 12, 87-97.

Wallbott, H.G., \& Scherer, K.R. (1994). Cultural determinants in experiencing shame and guilt. In June Price Tangney \& Kurt W. Fischer (Eds.), Self conscious emotions: The psychology of shame and guilt, embarrasment and pride (pp. 465-487). New York: Guilford Press. 